\title{
PROPERTIES OF CERTAIN SUBALGEBRAS OF DALES-DAVIE ALGEBRAS
}

\author{
M. ABTAHI and T. G. HONARY \\ Faculty of Mathematical Sciences and Computer Engineering, \\ Teacher Training University, Tehran, 15618, I.R. Iran \\ e-mails:m.abtahi@tmu.ac.ir,honary@saba.tmu.ac.ir
}

(Received 5 May, 2006; revised 24 September and 12 December, 2006; accepted 9 January, 2007)

\begin{abstract}
We study an interesting class of Banach function algebras of infinitely differentiable functions on perfect, compact plane sets. These algebras were introduced by H. G. Dales and A. M. Davie in 1973, called Dales-Davie algebras and denoted by $D(X, M)$, where $X$ is a perfect, compact plane set and $M=\left\{M_{n}\right\}_{n=0}^{\infty}$ is a sequence of positive numbers such that $M_{0}=1$ and $(m+n) ! / M_{m+n} \leq\left(m ! / M_{m}\right)\left(n ! / M_{n}\right)$ for $m, n \in$ N. Let $d=\lim \sup \left(n ! / M_{n}\right)^{1 / n}$ and $X_{d}=\{z \in \mathbf{C}: \operatorname{dist}(z, X) \leq d\}$. We show that, under certain conditions on $X$, every $f \in D(X, M)$ has an analytic extension to $X_{d}$. Let $D_{P}$ $\left[D_{R}\right]$ be the subalgebra of all $f \in D(X, M)$ that can be approximated by the restriction to $X$ of polynomials [rational functions with poles off $X$ ]. We show that the maximal ideal space of $D_{P}$ is $\widehat{X}_{d}$, the polynomial convex hull of $X_{d}$, and the maximal ideal space of $D_{R}$ is $X_{d}$. Using some formulae from combinatorial analysis, we find the maximal ideal space of certain subalgebras of Dales-Davie algebras.
\end{abstract}

2000 Mathematics Subject Classification. Primary 46J10, 46J15. Secondary 46J20.

1. Introduction. Let $X$ be a compact Hausdorff space. We denote the space of all continuous complex-valued functions on $X$ by $C(X)$. For $f \in C(X)$ and a closed subset $E$ of $X$, we denote the uniform norm of $f$ on $E$ by $|f|_{E}$. A function algebra on $X$ is a subalgebra $A$ of $C(X)$ that separates the points of $X$ and contains the constant functions. If there is an algebra norm on $A$ such that $A$ is complete under this norm, then $A$ is a Banach function algebra on $X$, and if the given norm is the uniform norm on $X$, then $A$ is a uniform algebra on $X$.

We denote by $\mathcal{M}(A)$, the maximal ideal space of $A$. Clearly, for each $x \in X$, the map $\varepsilon_{x}: A \rightarrow \mathbf{C}$, defined by $\varepsilon_{x}(f)=f(x)$, is a non-zero complex homomorphism on $A$ called the evaluation character at $x$. The map $J: X \rightarrow \mathcal{M}(A)$, defined by $J(x)=\varepsilon_{x}$, is injective and continuous, and so $X$ is homeomorphic to a compact subset of $\mathcal{M}(A)$. If the map $J$ is surjective, then $A$ is a natural Banach function algebra on $X$.

If $A$ is a Banach function algebra on $X$, then $\bar{A}$, the uniform closure of $A$ in $C(X)$, is a uniform algebra on $X$ and, clearly, $\mathcal{M}(\bar{A}) \subseteq \mathcal{M}(A)$. The following result in this area is proved in [5].

THEOREM 1.1. Let $X$ be a compact Hausdorff space and let $A$ be a Banach function algebra on $X$. Then $\mathcal{M}(A)=\mathcal{M}(\bar{A})$ if and only if $\left.\widehat{f}\right|_{\mathcal{M}(A)}=|f|_{X}$, for all $f \in A$, where $\widehat{f}$ is the Gelfand transform off.

For a compact plane set $X$, we denote the set of all functions on $X$ that are analytic on $\operatorname{int}(X)$ by $A(X)$, and the set of all functions on $X$ having an analytic 
extension to a neighborhood of $X$ by $H_{0}(X)$. We denote the set of the restriction to $X$ of rational functions with poles off $X$ by $R_{0}(X)$, and the set of the restriction to $X$ of polynomials by $P_{0}(X)$. The polynomial convex hull of $X$ is denoted by $\widehat{X}$. By the coordinate functional on $X$ we mean the function $z$ on $X$ that maps any point to itself.

Let $X$ be a perfect, compact plane set. We say that a complex-valued function $f$ on $X$ is complex-differentiable at a point $a \in X$ if the limit

$$
f^{\prime}(a)=\lim \left\{\frac{f(z)-f(a)}{z-a}, z \rightarrow a, z \in X\right\}
$$

exists. We call $f^{\prime}(a)$ the complex derivative of $f$ at $a$. We denote the $n$th derivative of $f$ at $a \in X$ by $f^{(n)}(a)$. We denote the set of $n$ times continuously complex-differentiable functions on $X$ by $D^{n}(X)$, and the set of infinitely complex-differentiable functions on $X$ by $D^{\infty}(X)$.

Let $M=\left\{M_{n}\right\}_{n=0}^{\infty}$ be a sequence of positive numbers such that $M_{0}=1$. Then $M$ is an algebra sequence if, for all $m, n \in \mathbf{N}$, we have

$$
\frac{(m+n) !}{M_{m+n}} \leq \frac{m !}{M_{m}} \frac{n !}{M_{n}} .
$$

$M$ is an analytic sequence if $d(M)=\limsup _{n \rightarrow \infty}\left(n ! / M_{n}\right)^{1 / n}>0$. If $d(M)=0$, the sequence $M$ is non-analytic.

For a perfect compact plane set $X$ and an algebra sequence $M=\left\{M_{n}\right\}_{n=0}^{\infty}$, a Dales-Davie algebra associated with $X$ and $M$ is defined by

$$
D(X, M)=\left\{f \in D^{\infty}(X): \sum_{k=0}^{\infty} \frac{\left|f^{(k)}\right|_{X}}{M_{k}}<\infty\right\},
$$

where the norm on $D(X, M)$ is given by

$$
\|f\|=\sum_{k=0}^{\infty} \frac{\left|f^{(k)}\right|_{X}}{M_{k}}
$$

Since $M$ is an algebra sequence, $D(X, M)$ is a normed function algebra on $X$.

A compact subset $X$ of the complex plane is connected by rectifiable arcs if any two points of $X$ can be joined by a rectifiable arc lying within $X$. For such a set, let $\delta(z, w)$ denote the geodesic distance between $z$ and $w$; that is, the infimum of the lengths of the arcs joining $z$ and $w$. Clearly $\delta$ defines a metric, the geodesic metric, on $X$.

DEFINITION 1.2. Let $X$ be a compact plane set which is connected by rectifiable arcs, and suppose that $\delta(z, w)$ is the geodesic distance between $z$ and $w$ in $X$.

(i) $X$ is regular if for any $z \in X$ there exists a constant $C_{z}$ such that $\delta(z, w) \leq$ $C_{z}|z-w|$, for all $w \in X$.

(ii) $X$ is uniformly regular if there exists a constant $C$ such that $\delta(z, w) \leq C|z-w|$, for all $z, w \in X$.

The following result is proved in [4, Lemma 1.5(iii)] for uniformly regular sets. An examination of the proof shows that it is also valid for regular sets. 
Lemma 1.3. Let $X$ be a regular set and let $z \in X$. Then, for all $f \in D^{n}(X)$ and every $\zeta \in X$

$$
\left|f(\zeta)-\sum_{k=0}^{n-1} \frac{f^{(k)}(z)}{k !}(\zeta-z)^{k}\right| \leq \frac{\left|f^{(n)}\right| X}{(n-1) !}\left(C_{z}\right)^{n}|\zeta-z|^{n}
$$

where $C_{z}$ is the constant in Definition 1.2.

Dales and Davie in [4] proved that if $X$ is a finite union of uniformly regular sets then $D(X, M)$ is complete. In fact, if $X$ is a finite union of regular sets then $D(X, M)$ is complete. For some further results see [2]. Throughout this note, we assume that $X$ is such a perfect compact plane set so that $D(X, M)$ is complete.

We need the following result about an algebra sequence $M$; see [3, Proposition A.1.26].

Lemma 1.4. Let $M=\left\{M_{n}\right\}_{n=0}^{\infty}$ be an algebra sequence. Then

$$
d(M)=\lim _{n \rightarrow \infty}\left(n ! / M_{n}\right)^{1 / n}=\inf \left\{\left(n ! / M_{n}\right)^{1 / n}: n \in \mathbf{N}\right\} .
$$

NotATION. Let $X$ be a compact plane set. For a point $\zeta \in \mathbf{C}$, the distance between $\zeta$ and $X$ is defined by $\operatorname{dist}(\zeta, X)=\inf \{|\zeta-z|: z \in X\}$. For a non-negative real number $d$, we set $X_{d}=\{\zeta \in \mathbf{C}: \operatorname{dist}(\zeta, X) \leq d\}$. If $d=0$ we set $\Omega_{d}=\operatorname{int}(X)$, and if $d>0$ we set $\Omega_{d}=\{\zeta \in \mathbf{C}: \operatorname{dist}(\zeta, X)<d\}$. For $z \in \mathbf{C}$ and $r>0, \mathbb{D}(z, r)=\{\zeta \in \mathbf{C}:|\zeta-z|<r\}$ and $\Delta(z, r)=\{\zeta \in \mathbf{C}:|\zeta-z| \leq r\}$, are the open and closed disc with center at $z$ and radius $r$.

2. Certain subalgebras. Clearly, for any algebra sequence $M=\left\{M_{n}\right\}_{n=0}^{\infty}$ and every perfect compact plane set $X, D(X, M)$ contains the polynomials on $X$; that is, $P_{0}(X) \subseteq$ $D(X, M)$.

Proposition 2.1. Let $d=d(M)$. Then $D(X, M)$ contains $H_{0}\left(X_{d}\right)$ and, moreover, the embedding of $H_{0}\left(X_{d}\right)$ in $D(X, M)$ is continuous in the sense that if $\left(f_{n}\right) \subseteq H_{0}\left(X_{d}\right)$ and $f_{n} \rightarrow f$ uniformly on a neighbourhood of $X_{d}$, then $f_{n} \rightarrow f$ in $D(X, M)$.

Proof. For $f \in H_{0}\left(X_{d}\right)$ there is a neighbourhood $U$ of $X_{d}$ such that $f$ is analytic on $U$. Choose $\rho>d$ so that $X_{\rho} \subseteq U$. By the Cauchy integral formula

$$
f^{(n)}(z)=\frac{n !}{2 \pi \mathrm{i}} \int_{C(z, \rho)} \frac{f(\zeta) \mathrm{d} \zeta}{(\zeta-z)^{n+1}} \quad(z \in X),
$$

where $C(z, \rho)$ is the circle with center at $z$ and radius $\rho$. Therefore,

$$
\frac{\left|f^{(n)}(z)\right|}{n !} \leq \frac{|f|_{X_{\rho}}}{2 \pi} \frac{2 \pi \rho}{\rho^{n+1}}=\frac{|f|_{X_{\rho}}}{\rho^{n}}
$$

and

$$
\|f\|=\sum_{n=0}^{\infty} \frac{\left|f^{(n)}\right|_{X}}{M_{n}} \leq|f|_{X_{\rho}} \sum_{n=0}^{\infty} \frac{1}{\rho^{n}} \frac{n !}{M_{n}}<\infty
$$


Now, suppose that $f_{n} \rightarrow f$ uniformly in some neighbourhood of $X_{d}$. We can choose $\rho>d$ such that $\left|f_{n}-f\right|_{X_{\rho}} \rightarrow 0$. Similarly, we have

$$
\lim _{n \rightarrow \infty}\left\|f_{n}-f\right\| \leq \lim _{n \rightarrow \infty}\left|f_{n}-f\right|_{X_{\rho}} \sum_{m=0}^{\infty} \frac{1}{\rho^{m}} \frac{m !}{M_{m}}=0 .
$$

Proposition 2.2. Suppose that $d$ is a non-negative real number. Then $D(X, M)$ contains $R_{0}\left(X_{d}\right)$ if and only if $d(M) \leq d$.

Proof. If $d(M) \leq d$ then, by Proposition $2.1, R_{0}\left(X_{d}\right) \subseteq D(X, M)$. Suppose that $R_{0}\left(X_{d}\right) \subseteq D(X, M)$. Let $\rho>d$ be arbitrary, and let $\zeta \in \mathbf{C}$ be such that $\rho=\operatorname{dist}(\zeta, X)$. By the assumption, if $f(z)=1 /(\zeta-z)$ for $z \neq \zeta$, then $f \in D(X, M)$. Therefore

$$
\|f\|=\sum_{n=0}^{\infty} \frac{\left|f^{(n)}\right|_{X}}{M_{n}}=\sum_{n=0}^{\infty} \frac{n !}{M_{n}} \frac{1}{\rho^{n+1}}<\infty .
$$

This implies that $d(M)=\lim \sup _{n \rightarrow \infty}\left(n ! / M_{n}\right)^{1 / n} \leq \rho$ and, since $\rho>d$ is arbitrary, we have $d(M) \leq d$.

Corollary 2.3. $R_{0}(X) \subseteq D(X, M)$ if and only if $\lim _{n \rightarrow \infty}\left(n ! / M_{n}\right)^{1 / n}=0$.

Definition 2.4. Let $d=d(M)$. We define $D_{P}(X, M), D_{R}(X, M)$, and $D_{H}(X, M)$ to be the closure of $P_{0}\left(X_{d}\right), R_{0}\left(X_{d}\right)$ and $H_{0}\left(X_{d}\right)$ in $D(X, M)$, respectively. These algebra are simply denoted by $D_{P}, D_{R}$, and $D_{H}$.

Corollary 2.5. Let $d=d(M)$. Then

(i) $H_{0}\left(\widehat{X}_{d}\right) \subseteq D_{P}$,

(ii) $H_{0}\left(X_{d}\right) \subseteq D_{R}$ and, consequently, $D_{R}=D_{H}$.

Proof. For $f \in H_{0}\left(\widehat{X}_{d}\right)$, there is $\rho>d$ such that $f \in H_{0}\left(\widehat{X}_{\rho}\right)$. By Runge's theorem, there is a sequence $\left(p_{n}\right)$ of polynomials such that $\left|p_{n}-f\right|_{X_{\rho}} \rightarrow 0$ as $n \rightarrow \infty$. By (2.1),

$$
\left\|p_{n}-f\right\| \leq\left|p_{n}-f\right|_{X_{\rho}} \sum_{n=0}^{\infty} \frac{1}{\rho^{n}} \frac{n !}{M_{n}} \rightarrow 0 .
$$

The proof of part (ii) is similar.

3. Extension of infinitely differentiable functions. Throughout this section, we assume that $M=\left\{M_{n}\right\}_{n=0}^{\infty}$ is an analytic algebra sequence with $d=d(M)>0$, and we write $D$ for $D(X, M)$. For $f \in D$ and for $\zeta \in X$, by Lemma 1.4, we have that

$$
F_{z}(\zeta)=\sum_{k=0}^{\infty} \frac{f^{(k)}(z)}{k !}(\zeta-z)^{k}
$$

is uniformly convergent in $\Delta(z, d)$, and so the function $F_{z}: \Delta(z, d) \rightarrow \mathbf{C}$ is continuous on $\Delta(z, d)$ and analytic on $\mathbb{D}(z, d)$. A natural question arises here.

Does $f \in D$ have an extension in $A\left(X_{d}\right)$ ? 
It seems that the answer to this question depends on $X$. Dales in [3, Theorem 4.4.16 (i)] has answered this question for $X=[0,1]$ as follows.

THEOREM 3.1. Let $X=[0,1]$, and let $M$ be an analytic algebra sequence. Then $D$ is isomorphic to a natural Banach function algebra $\widehat{D}$ on $X_{d}$, and $\widehat{D}$ is contained in $A\left(X_{d}\right)$.

We answer this question in more general cases.

THEOREM 3.2. Let $X$ be a perfect, compact plane set. Then every $f \in D_{H}$ has a unique extension $F$ in $A\left(X_{d}\right)$ and $|F|_{X_{d}} \leq\|f\|$.

Proof. Let $f \in D_{H}$. There is a sequence $\left\{f_{n}\right\}$ in $H_{0}\left(X_{d}\right)$ such that $\left\|f_{n}-f\right\| \rightarrow 0$. In particular, $\left\{f_{n}\right\}$ is a Cauchy sequence in $D$. We show that $\left\{f_{n}\right\}$ is a Cauchy sequence in $C\left(X_{d}\right)$. Let $\zeta \in X_{d}$. Then there is $z \in X$ such that $|\zeta-z| \leq d$. Since every $f_{n}$ is analytic in a neighborhood of $X_{d}$, we have

$$
f_{n}(\zeta)=\sum_{k=0}^{\infty} \frac{f_{n}^{(k)}(z)}{k !}(\zeta-z)^{k} \quad(n \in \mathbf{N})
$$

Consequently

$$
\begin{aligned}
\left|f_{n}(\zeta)-f_{m}(\zeta)\right| & \leq \sum_{k=0}^{\infty} \frac{\left|f_{n}^{(k)}-f_{m}^{(k)}\right|_{X}}{k !}|\zeta-z|^{k} \\
& \leq \sum_{k=0}^{\infty} \frac{\left|f_{n}^{(k)}-f_{m}^{(k)}\right|_{X}}{M_{k}} \frac{M_{k}}{k !} d^{k} \\
& \leq\left\|f_{n}-f_{m}\right\| .
\end{aligned}
$$

This shows that $\left|f_{n}-f_{m}\right|_{X_{d}} \rightarrow 0$ as $m, n \rightarrow \infty$. Let $F \in C\left(X_{d}\right)$ be the uniform limit of $\left\{f_{n}\right\}$ on $X_{d}$; then $F \in A\left(X_{d}\right),\left.F\right|_{X}=f$, and $|F|_{X_{d}}=\lim _{n \rightarrow \infty}\left|f_{n}\right|_{X_{d}} \leq \lim _{n \rightarrow \infty}\left\|f_{n}\right\|=\|f\|$.

Suppose that $F_{1} \in A\left(X_{d}\right)$ and $\left.F_{1}\right|_{X}=f$. Since $X$ is perfect, it has a limit point and this implies that $F_{1}=F$.

Corollary 3.3. Let $X$ be a perfect, compact plane set. Then every $f \in D_{P}(X, M)$ has a unique extension $F$ in $A\left(\widehat{X}_{d}\right)$ and $|F|_{\widehat{X}_{d}} \leq\|f\|$.

Proof. Let $f \in D_{P}$. There is a sequence $\left\{p_{n}\right\}$ of polynomials such that $\left\|p_{n}-f\right\| \rightarrow$ 0 . As in the proof of Theorem 3.2, $\left|p_{m}-p_{n}\right|_{X_{d}} \rightarrow 0$ as $m, n \rightarrow \infty$, so that $\left|p_{m}-p_{n}\right|_{\widehat{X}_{d}} \rightarrow$ 0 as $m, n \rightarrow \infty$. Let $F \in C\left(\widehat{X}_{d}\right)$ be the uniform limit of $\left\{p_{n}\right\}$ on $\widehat{X}_{d}$. Clearly $F \in$ $A\left(\widehat{X}_{d}\right),\left.F\right|_{X}=f$ and $|F|_{\widehat{X}_{d}}=\lim _{n \rightarrow \infty}\left|p_{n}\right|_{\widehat{X}_{d}}=\lim _{n \rightarrow \infty}\left|p_{n}\right|_{X_{d}} \leq \lim _{n \rightarrow \infty}\left\|p_{n}\right\|=\|f\|$. The uniqueness of $F$ follows from Theorem 3.2.

In the rest of this section, we try to find sufficient conditions on $X$ to guarantee that every $f \in D$ can be extended to a function $F$ in $A\left(X_{d}\right)$.

Lemma 3.4. Suppose that $X$ is regular and $f \in D$. Then for every $z \in X$, there exists $r=r(z)>0$ such that $f(\zeta)=F_{z}(\zeta)$ for $\zeta \in \mathbb{D}(z, r) \cap X$. In particular, $f$ has an analytic extension on $\mathbb{D}(z, r)$. 
Proof. Let $z \in X$, and let $r=d / C_{z}$, where $C_{z}$ is the constant in Definition 1.2. If $\zeta \in X$, and $|\zeta-z|<r$, then for some $\beta>1,|\zeta-z| \leq d /\left(\beta C_{z}\right)$. By Lemma 1.3,

$$
\begin{aligned}
\lim _{n \rightarrow \infty}\left|f(\zeta)-\sum_{m=0}^{n-1} \frac{f^{(k)}(z)}{k !}(\zeta-z)^{k}\right| & \leq \lim _{n \rightarrow \infty}\left(\frac{\left|f^{(n)}\right|_{X}}{(n-1) !}\left(C_{z}\right)^{n}|\zeta-z|^{n}\right) \\
& \leq \lim _{n \rightarrow \infty}\left(\frac{\left|f^{(n)}\right|_{X}}{(n-1) !}(d / \beta)^{n}\right)=0
\end{aligned}
$$

This shows that $f(\zeta)=F_{z}(\zeta)$, for all $\zeta \in \mathbb{D}(z, r) \cap X$.

COROLlary 3.5. Let $X$ be a regular set. Then $D \subset H_{0}(X)$.

Proof. Let $f \in D$. By Lemma 3.4, since $X$ is regular, for each $z \in X$, there is $r(z)>0$ such that $f=F_{z}$ on $\mathbb{D}(z, r(z)) \cap X$. There exist $z_{1}, \ldots, z_{n} \in X$ such that $X \subseteq$ $U=\bigcup_{j=1}^{n} \mathbb{D}\left(z_{j}, r_{j} / 2\right)$, where $r_{j}=r\left(z_{j}\right)$. Define $F: U \rightarrow \mathbf{C}$ by $F(\zeta)=F_{z_{j}}(\zeta)$, where $\zeta \in$ $\mathbb{D}\left(z_{j}, r_{j} / 2\right)$. Suppose that $\mathbb{D}\left(z_{i}, r_{i} / 2\right) \cap \mathbb{D}\left(z_{j}, r_{j} / 2\right) \neq \varnothing$, for some $i, j$. Then $\left|z_{i}-z_{j}\right|<$ $\max \left\{r_{i}, r_{j}\right\}$ and, therefore, $E=\mathbb{D}\left(z_{i}, r_{i}\right) \cap \mathbb{D}\left(z_{j}, r_{j}\right) \cap X \neq \varnothing$. Since $F_{z_{i}}=F_{z_{j}}$ on $E$ and $E$ has a limit point, $F_{z_{i}}=F_{z_{j}}$ on $\mathbb{D}\left(z_{i}, r_{i} / 2\right) \cap \mathbb{D}\left(z_{j}, r_{j} / 2\right)$. Hence, $F$ is an analytic extension of $f$ on $U$, and so $f \in H_{0}(X)$.

COROLlaRY 3.6. Let $X$ be a regular set and $a \in X$. Suppose that $f \in D$ and $f^{(k)}(a)=$ 0 , for $k \geq 0$. Then $f=0$.

THEOREM 3.7. Let $X$ be a regular set, and let $Q=\{(z, w) \in X \times X:|z-w|<2 d\}$. If $Q$ is connected then every $f \in D$ has an analytic extension to $\Omega_{d}$. Moreover

$$
\sup \left\{|F(\zeta)|: \zeta \in \Omega_{d}\right\} \leq\|f\|
$$

Proof. Define $S=\left\{(z, w) \in Q: F_{z}(\zeta)=F_{w}(\zeta)\right.$, for all $\left.\zeta \in \mathbb{D}(z, d) \cap \mathbb{D}(w, d)\right\}$. We show that $S$ is an open and closed subset of $Q$.

Let $(z, w) \in S$. By Lemma 3.4, there exist positive numbers $r(z)$ and $r(w)$ such that $F_{z}=f$ on $\mathbb{D}(z, r(z)) \cap X, F_{w}=f$ on $\mathbb{D}(w, r(w)) \cap X$, and moreover, if $z_{1} \in$ $\mathbb{D}(z, r(z))$ and $w_{1} \in \mathbb{D}(w, r(w))$ then $\mathbb{D}(z, d) \cap \mathbb{D}(w, d) \cap \mathbb{D}\left(z_{1}, d\right) \cap \mathbb{D}\left(w_{1}, d\right) \neq \varnothing$. Set $V=\mathbb{D}(z, r(z)) \times \mathbb{D}(w, r(w))$, and let $\left(z_{1}, w_{1}\right) \in V \cap Q$. Then $F_{z_{1}}=F_{z}=f$ on $\mathbb{D}(z, r(z)) \cap$ $X$ and $F_{w_{1}}=F_{w}=f$ on $\mathbb{D}(w, r(w)) \cap X$. Thus $F_{z_{1}}=F_{z}$ on $\mathbb{D}(z, d) \cap \mathbb{D}\left(z_{1}, d\right) \cap X$ and $F_{w_{1}}=F_{w}$ on $\mathbb{D}(w, d) \cap \mathbb{D}\left(w_{1}, d\right) \cap X$. This shows that $F_{z_{1}}=F_{w_{1}}$ on $\mathbb{D}\left(z_{1}, d\right) \cap$ $\mathbb{D}\left(w_{1}, d\right) \cap X, V \cap Q \subseteq S$ and, therefore, $S$ is an open subset of $Q$.

We now show that $S$ is a closed subset of $Q$. Let $(z, w) \in Q \backslash S$, and let $V$ be the neighborhood of $(z, w)$ given in the proof of openness of $S$ in the last paragraph. Let $\left(z_{1}, w_{1}\right) \in V \cap Q$. Using the same argument, if $F_{z_{1}}=F_{w_{1}}$ then $F_{z}=F_{w}$. Since $(z, w) \in Q \backslash S, F_{z} \neq F_{w}$, and so $F_{z_{1}} \neq F_{w_{1}}$. This shows that $V \cap Q \subseteq Q \backslash S$, and thus $Q \backslash S$ is open in $Q$.

Therefore, $S$ is a non-empty closed and open subset of $Q$ and, since $Q$ is connected, we conclude that $S=Q$, and hence $F$ is the desired extension of $f$ to $\Omega_{d}$.

We do not completely know for which $X$ every $f \in D$ can be extended to a function in $A\left(X_{d}\right)$. One may think that if $X$ is a set with the property that each pair $z, w$ of points in $X$ can be joined by a sufficiently smooth curve within $X$, then every $f \in D$ 
has an analytic extension on $\Omega_{d}$. The following example shows that this is not true in general.

ExAmple. For $\varepsilon>0$ small, let $X=\left\{2 e^{\mathrm{i} \theta}:-\pi+\varepsilon \leq \theta \leq \pi-\varepsilon\right\}$. Suppose that $M_{n}=n !$ and

$$
f(z)=\log (z)=\ln |z|+\mathrm{i} \theta \quad(-\pi<\theta<\pi) .
$$

Then $f \in D$ and $d=1$. Clearly, for $\varepsilon$ small enough, $f$ has no analytic extension on $\Omega_{d}$.

4. Maximal ideal space. Dales and Davie in [4] proved that if $d(M)=0$, then $\mathcal{M}\left(D_{R}\right)=X$ and $\mathcal{M}\left(D_{P}\right)=\widehat{X}$. We have the following result.

THEOREM 4.1. Let $X$ be a compact, perfect plane set such that $D(X, M)$ is complete. Then $\mathcal{M}\left(D_{R}\right)=X_{d}$ and $\mathcal{M}\left(D_{P}\right)=\widehat{X}_{d}$, where $d=d(M)$.

Proof. Let $\zeta \in X_{d}$. We define $h_{\zeta}: D_{R} \rightarrow \mathbf{C}$ by $h_{\zeta}(f)=F(\zeta)$, where $F$ is the unique extension of $f$ in $A\left(X_{d}\right)$ given by Theorem 3.2. Theorem 3.2 shows that $h_{\zeta} \in \mathcal{M}\left(D_{R}\right)$.

Let $h \in \mathcal{M}\left(D_{R}\right)$ and $\zeta=h(z)$, where $z$ is the coordinate functional on $X$. If $\zeta \notin X_{d}$, then $\frac{1}{\zeta-z} \in D_{R}$ which is a contradiction, and so $\zeta \in X_{d}$. It is easy to see that $h(f)=f(\zeta)$, for all $f \in R_{0}\left(X_{d}\right)$. For $f \in D_{R}$, there is a sequence $\left\{f_{n}\right\}$ in $R_{0}\left(X_{d}\right)$ such that $\left\|f_{n}-f\right\| \rightarrow 0$. Again, by Theorem 3.2, $f_{n}(\zeta) \rightarrow f(\zeta)$ and therefore $h(f)=\lim _{n \rightarrow \infty} h\left(f_{n}\right)=\lim _{n \rightarrow \infty} f_{n}(\zeta)=f(\zeta)$.

Similarly, for $\zeta \in \widehat{X}_{d}$, define $h_{\zeta}: D_{P} \rightarrow \mathbf{C}$ by $h_{\zeta}(f)=F(\zeta)$, where $F$ is the unique extension of $f$ in $A\left(\widehat{X}_{d}\right)$ given by Corollary 3.3. By Corollary 3.3, $h_{\zeta} \in \mathcal{M}\left(D_{P}\right)$.

Let $h \in \mathcal{M}\left(D_{P}\right)$, and $\zeta=h(z)$, where $z$ is the coordinate functional on $X$. If $\zeta \notin \widehat{X}_{d}$ then $\frac{1}{\zeta-z} \in D_{P}$, which is a contradiction, so that $\zeta \in \widehat{X}_{d}$. It is easy to see that $h(p)=$ $p(\zeta)$ for all polynomials $p$. For $f \in D_{P}$, there is a sequence $\left\{p_{n}\right\}$ of polynomials such that $\left\|p_{n}-f\right\| \rightarrow 0$. Again, by Corollary $3.3, p_{n}(\zeta) \rightarrow f(\zeta)$ and $h(f)=\lim _{n \rightarrow \infty} h\left(p_{n}\right)=$ $\lim _{n \rightarrow \infty} p_{n}(\zeta)=f(\zeta)$

To continue we need some formulae from combinatorial analysis. For $m, n \in \mathbf{N}$ with $n \geq m$, we take $S(m, n)$ as the set of all $\left(a_{1}, a_{2}, \ldots, a_{n}\right) \in\left(\mathbf{Z}^{+}\right)^{n}$ such that $a_{1}+$ $a_{2}+\cdots+a_{n}=m$ and $a_{1}+2 a_{2}+\cdots+n a_{n}=n$. For any $m \in \mathbf{N}$ and any sequence $\left\{A_{k}\right\}$ of positive numbers, by [1, Formula B3, p. 823],

$$
\left(\sum_{k=1}^{\infty} A_{k}\right)^{m}=m ! \sum_{n=m}^{\infty} \sum \frac{1}{\prod a_{k} !} \prod_{k=1}^{n}\left(A_{k}\right)^{a_{k}},
$$

where the inner sum is taken over all $\left(a_{1}, a_{2}, \ldots, a_{n}\right) \in S(m, n)$. The following equality for higher derivatives of composite functions is known as the Faa di Bruno's Formula (see [1, p. 823]):

$$
(F \circ f)^{(n)}=\sum_{m=0}^{n} F^{(m)}(f) \sum \frac{n !}{\prod a_{k} !} \prod_{k=1}^{n}\left(\frac{f^{(k)}}{k !}\right)^{a_{k}},
$$

where the inner sum is taken over all $\left(a_{1}, a_{2}, \ldots, a_{n}\right) \in S(m, n)$. 
LEMMA 4.2. Let $K>0$ and $\left\{\varepsilon_{m}\right\}$ be a sequence of positive numbers such that $\varepsilon_{m} \rightarrow 0$ as $m \rightarrow \infty$. Then

$$
\underset{p \rightarrow \infty}{\limsup }\left(\sum_{m=0}^{p}\left(\begin{array}{l}
p \\
m
\end{array}\right)\left(\varepsilon_{m}\right)^{m} K^{p-m}\right)^{1 / p} \leq K .
$$

Proof. Without loss of generality, we may assume that $K=1$. For $\varepsilon>0$ there is $N \in \mathbb{N}$ such that $\varepsilon_{m}<\varepsilon$, for any $m>N$. For $p>N$ we have

$$
\begin{aligned}
\sum_{m=0}^{p}\left(\begin{array}{l}
p \\
m
\end{array}\right)\left(\varepsilon_{m}\right)^{m} & \leq \sum_{m=0}^{N}\left(\begin{array}{l}
p \\
m
\end{array}\right)\left(\varepsilon_{m}\right)^{m}+\sum_{m=N+1}^{p}\left(\begin{array}{l}
p \\
m
\end{array}\right) \varepsilon^{m} \\
& \leq p^{N} \sum_{m=0}^{N}\left(\varepsilon_{m}\right)^{m}+(1+\varepsilon)^{p} \\
& \leq 2 p^{N}(1+\varepsilon)^{p} \sum_{m=0}^{N}\left(\varepsilon_{m}\right)^{m}
\end{aligned}
$$

Hence $\lim \sup _{p \rightarrow \infty}\left(\sum_{m=0}^{p}\left(\begin{array}{l}p \\ m\end{array}\right)\left(\varepsilon_{m}\right)^{m}\right)^{1 / p} \leq 1+\varepsilon$, which implies the required in equality.

Lemma 4.3. Let $X$ be a perfect compact plane set, and let $M=\left\{M_{n}\right\}_{n=0}^{\infty}$ be an algebra sequence. Suppose that $A=\left\{f \in D^{\infty}(X): f^{\prime} \in D(X, M)\right\}$.

(i) The set $A$ is a subalgebra of $D(X, M)$.

(ii) If $d(M)=0$, then $|f|_{X}=|\widehat{f}|_{\mathcal{M}(D)}$ for all $f \in A$.

Proof. (i) Let $f \in A$. Since $\frac{n+1}{M_{n+1}} \leq \frac{1}{M_{1} M_{n}}$, we have

$$
\sum_{n=0}^{\infty} \frac{\left|f^{(n+1)}\right|_{X}}{M_{n+1}} \leq \sum_{n=0}^{\infty} \frac{\left|f^{(n+1)}\right|_{X}}{M_{n}} \frac{1}{(n+1) M_{1}} \leq \sum_{n=0}^{\infty} \frac{\left|\left(f^{\prime}\right)^{(n)}\right|_{X}}{M_{n}}<\infty
$$

Hence $f \in D(X, M)$, and $A \subseteq D(X, M)$. It is easy to see that $A$ is, in fact, a subalgebra of $D(X, M)$.

(ii) Let $f \in A$. For $p \in \mathbf{N}$, by the Faa di Bruno's formula (4.2) we have

$$
\left\|f^{p}\right\|=\sum_{n=0}^{\infty} \frac{\left|\left(f^{p}\right)^{(n)}\right|_{X}}{M_{n}} \leq \sum_{n=0}^{\infty} \sum_{m=0}^{p} m !\left(\begin{array}{c}
p \\
m
\end{array}\right) \frac{|f|_{X}^{p-m}}{M_{n}} \sum \frac{n !}{\prod a_{k} !} \prod_{k=1}^{n}\left(\frac{\left|f^{(k)}\right|_{X}}{k !}\right)^{a_{k}} .
$$

After interchanging the order of summation, we have

$$
\begin{aligned}
\left\|f^{p}\right\| & \leq \sum_{m=0}^{p}\left(\begin{array}{c}
p \\
m
\end{array}\right)|f|_{X}^{p-m} m ! \sum_{n=m}^{\infty} \sum \frac{1}{\prod a_{k} !} \frac{\prod_{k=1}^{n}\left(P_{k-1}\right)^{a_{k}}}{P_{n}} \prod\left(\frac{\left|f^{(k)}\right|_{X}}{k M_{k-1}}\right)^{a_{k}} \\
& \leq \sum_{m=0}^{p}\left(\begin{array}{c}
p \\
m
\end{array}\right)|f|_{X}^{p-m} m ! \sum_{n=m}^{\infty} \sum \frac{1}{\prod a_{k} !} \frac{P_{n-m}}{P_{n}} \prod\left(\frac{\left|f^{(k)}\right|_{X}}{k M_{k-1}}\right)^{a_{k}} \\
& \leq \sum_{m=0}^{p}\left(\begin{array}{c}
p \\
m
\end{array}\right)|f|_{X}^{p-m} \frac{m !}{P_{m}} \sum_{n=m}^{\infty} \sum \frac{1}{\prod a_{k} !} \prod\left(\frac{\left|f^{(k)}\right|_{X}}{M_{k-1}}\right)^{a_{k}} .
\end{aligned}
$$


By Formula (4.1),

$$
\left\|f^{p}\right\| \leq \sum_{m=0}^{p}\left(\begin{array}{c}
p \\
m
\end{array}\right)|f|_{X}^{p-m}\left(\left(1 / P_{m}\right)^{1 / m}\left\|f^{\prime}\right\|\right)^{m} .
$$

Applying Lemma 4.2, with $\varepsilon_{m}=\left\|f^{\prime}\right\|\left(m ! / M_{m}\right)^{1 / m}$, we have $\left.\widehat{\mid f}\right|_{\mathcal{M}(D)} \leq|f|_{X}$.

THEOREM 4.4. Let $M=\left\{M_{n}\right\}_{n=0}^{\infty}$ be a non-analytic algebra sequence $(d(M)=0)$, and let $X$ be uniformly regular. Then $B=\bar{A}$, the closure of $A$ in $D(X, M)$, is a natural subalgebra of $D(X, M)$.

Proof. Since $d(M)=0, R_{0}(X) \subseteq B \subseteq D^{1}(X)$. By [4, Lemma 1.5(iv)], $D^{1}(X) \subseteq$ $R(X)$. This shows that $\bar{B}=R(X)$, where $\bar{B}$ is the uniform closure of $B$ in $C(X)$. Using Theorem 1.1, it is enough to show that $\left.\widehat{f}\right|_{\mathcal{M}(D)}=|f|_{X}$, for all $f \in B$.

Let $f \in B$. Then there exists a sequence $\left\{f_{n}\right\}$ in $A$ such that $\left\|f_{n}-f\right\| \rightarrow 0$ as $n \rightarrow \infty$. Since $\left|f_{n}-f\right|_{X} \leq \widehat{\mid f_{n}}-\left.\widehat{f}\right|_{\mathcal{M}(D)} \leq\left\|f_{n}-f\right\|$, we conclude that $\left|\widehat{f}_{n}\right|_{\mathcal{M}(D)} \rightarrow|\widehat{f}|_{\mathcal{M}(D)}$ and $\left|f_{n}\right|_{X} \rightarrow|f|_{X}$. By the previous result, $\left|\widehat{f}_{n}\right|_{\mathcal{M}(D)}=\left|f_{n}\right|_{X}$ and so $\left.\widehat{\mid f}\right|_{\mathcal{M}(D)}=|f|_{X}$.

Question. Is the subalgebra $A=\left\{f \in D^{\infty}(X): f^{\prime} \in D(X, M)\right\}$ dense in $D(X, M)$ ?

ACKnOwledgment. Part of this work was done when the first author was visiting the Department of Mathematics at the University of Manitoba. He wishes to express his gratitude for its hospitality during his stay. He also would like to sincerely express his appreciation to Professor Y. Zhang for his invaluable comments.

\section{REFERENCES}

1. M. Abramowitz and I. Stegun, Handbook of mathematical functions with formulas, graphs and mathematical tables (U.S. Department of Commerce, Washington, D.C., 1964).

2. W. J. Bland and J. F. Feinestein, Completion of normed algebras of differentiable functions, Studia Mathematica 170 (2005), 89-111.

3. H. G. Dales, Banach algebras and automatic continuity, London Mathematical Society Monographs, New Series No. 24 (Oxford University Press).

4. H. G. Dales and A. M. Davie, Quasi-analytic Banach function algebras, J. Functional Analysis 13 (1973), 28-50.

5. T. G. Honary, Relations between Banach function algebras and their uniform closusres, Proc. Amer. Math. Soc. 109 (1990), 337-342. 